\title{
The effect of musical training on music processing: a functional magnetic resonance imaging study in humans
}

\author{
Vincent J. Schmithorst*, Scott K. Holland \\ Imaging Research Center, Children's Hospital Medical Center, Mail Location 5031, 3333 Burnet Avenue, Cincinnati, OH 45229, USA
}

Received 14 April 2003; received in revised form 29 May 2003; accepted 29 May 2003

\begin{abstract}
Previous studies have demonstrated changes in neuronal activity in trained musicians relative to controls while performing various music processing tasks. In this study the neural correlates of the effect of music training on two aspects of music processing, melody and harmony, are investigated using functional magnetic resonance imaging (fMRI). Fifteen subjects, seven with continuous musical training from early childhood to adulthood and eight without, underwent a passive fMRI listening paradigm designed to test the effects of melodic and harmonic processing. Melodic processing activated the most anterior part of the superior temporal gyrus for both musicians and non-musicians, while harmonic processing activated different visual association areas for musicians relative to non-musicians. The inferior parietal lobules were recruited only by musicians for both tasks. We conclude that musical training results in the recruitment of different neural networks for these aspects of music processing.
\end{abstract}

(C) 2003 Elsevier Ireland Ltd. All rights reserved.

Keywords: Music; Functional magnetic resonance imaging; Adult; Human; Cognition; Auditory perception; Pitch perception

Formal musical training has been found to have an effect on the neural representation of various elements of music processing. Musicians displayed increased event-related potentials (ERPs) relative to non-musicians when listening to occasionally omitted sounds in a regular tone series [11] and during a timbre discrimination task [3]. In an electroencephalogram (EEG) study [2], increased phase synchrony was found in musicians while listening to music as compared to normal controls. In this study the neural correlates of the effects of music training on two specific elements of music processing, melody and harmony, are investigated using functional magnetic resonance imaging (fMRI). FMRI enables the detection of region-specific differences in neuronal activity and allows for the testing of the hypothesis that extensive musical training will result in the recruitment of different neural networks and hence produce different activation patterns in musicians relative to non-musicians.

Fifteen normal adult volunteers $(4 \mathrm{~F}, 11 \mathrm{M}$, average age $37.8 \pm 15.2$ years) participated in the study. Informed consent and Institutional Review Board approval were

\footnotetext{
* Corresponding author. Tel.: + 1-513-636-3922; fax: + 1-513-636-3754.

E-mail address: vince@athena.chmcc.org (V.J. Schmithorst).
}

obtained for all subjects. We used as our criterion for classifying a subject as a 'musician' that he or she have engaged in musical study continuously from early childhood (age 8 or before) to late adolescence. Seven of the subjects met this criterion and were classified as musicians, while eight did not.

A three-phase block-design fMRI paradigm was used. The subjects were presented with $30 \mathrm{~s}$ of an unharmonized popular melody, followed by $30 \mathrm{~s}$ of tones of random frequency and duration, followed by $30 \mathrm{~s}$ of the previous melody, harmonized using triads an octave below. The frequency range of the random tones was matched to the frequency range of the harmonized melodies. Pure sine tones were used throughout. An Audio Interchange File Format (AIFF) sound file was generated in IDL (Interactive Data Language, Research Systems Inc., Boulder, CO) and played on an SGI O2 (Silicon Graphics Inc., Silicon Valley, CA) computer system through an MRI-compatible audio/video system (Magnetic Resonance Technologies, Van Nuys, CA). The volume was adjusted to ensure that the subjects could hear the stimuli clearly over the MRI scanner noise.

MRI images were obtained using a 3T Bruker Biospec system (Bruker Medical Instruments, Karlsruhe, Germany). 
For the functional imaging scans, a 24-slice blipped echoplanar imaging (EPI) sequence was used with the following parameters: matrix $=64 \times 64$, bandwidth $=125 \mathrm{kHz}$, field of view $=25.6 \times 25.6 \mathrm{~cm}$, echo time $(\mathrm{TE})=38 \mathrm{~ms}$, repetition time $(\mathrm{TR})=3 \mathrm{~s}$, slice thickness $=5 \mathrm{~mm}$. Fifteen seconds of EPI images were acquired prior to the beginning of the paradigm in order to allow the spins to reach T1 relaxation equilibrium, followed by five sets of unharmonized melodies, random tones, and harmonized melodies, for a total scan time of $7 \mathrm{~min} 45 \mathrm{~s}$. In addition, a whole-brain T1-weighted anatomical scan was acquired for anatomical coregistration.

FMRI post-processing was performed in IDL. The geometrical distortion and Nyquist ghost artifacts were corrected for via the multi-echo reference technique [13], and a Hamming filter was applied to the raw EPI data prior to reconstruction in order to reduce high-frequency noise. The images were corrected for motion using a pyramid iterative algorithm [16], and transformed into stereotaxic coordinates [15] using landmarks found from the wholebrain anatomical images. The General Linear Model [17] was used in order to perform the contrasts of unharmonized melodies vs. random tones and harmonized melodies vs. unharmonized melodies, in order to investigate the effects of melodic and harmonic processing, respectively. A set of cosine basis functions was used as covariates to account for possible signal drift and aliased respiratory and cardiac signals. Z-Score maps were computed and then smoothed in three dimensions with a Gaussian filter of width $4 \mathrm{~mm}$. A random-effects analysis [4] was performed in order to define regions of significant activation for each group (musicians and non-musicians), and the clustering method [18] was used in order to increase specificity. A Monte Carlo simulation revealed that the thresholding criteria used (nominal $P<0.01$, cluster size $=9$ ) resulted in a corrected $P /$ cluster of $<0.001$. The subset of voxels determined to be active in either group were retained for further analysis. A random-effects analysis was again performed in order to detect group differences, with a Monte Carlo simulation revealing that the thresholding criteria used (nominal $P<0.05$, cluster size $=7$ ) resulted in a corrected $P /$ cluster of $<0.05$.

For the contrast of unharmonized melodies vs. random tones, statistically significantly greater activation in musicians was found in the inferior parietal lobules (BA 40) and superior frontal gyrus bilaterally, and the left inferior frontal and superior temporal gyri. The most anterior portion of the superior temporal gyrus was activated bilaterally in both musicians and non-musicians.

For the contrast of harmonized melodies vs. unharmonized melodies, statistically significant group differences were found in the right lingual gyrus and the left inferior parietal lobule (BA 39) (musicians > non-musicians), and the right fusiform gyrus, left medial occipital gyrus, left inferior frontal gyrus, left superior frontal gyrus, and anterior cingulate (musicians $<$ non-musicians).
The robust activation found in the anterior part of the superior temporal gyrus for the melodic processing task (Fig. 1) is in line with previous results [8] implicating the superior temporal gyrus in melodic processing, and has also been hypothesized [9] as being involved in the processing of non-verbal semantic information. Our results lend support to that hypothesis, and indicate that the capacity for melodic processing which exists in the superior temporal gyrus is one which does not require specialized musical training to develop.

The increased activation in musicians found in the left inferior frontal and superior temporal gyri agree with previously reported results [9] implicating those areas in the semantic familiarity of melodies. The superior frontal gyrus and inferior parietal lobule were also reported as activated during a pitch judgment task [9], where subjects were asked to compare two melodies to determine if any of the pitches were different, and it was hypothesized that a visual mental strategy was employed in the processing of the intervals. While further research will be necessary to corroborate this hypothesis, we note that our results are in line with the expectation that trained musicians would employ intervallic analysis techniques to a greater degree than non-musicians.

For harmonic processing, the activation found in visual association areas for both musicians and non-musicians may indicate that, even without musical training, exposure to harmonic progressions integrates with visual associative functions in the brain. Occipital lobe activation was found during a previous study in which subjects were instructed to listen specifically to the harmony [12]. The shift in activation patterns from the right fusiform gyrus to the lingual gyrus bilaterally (Fig. 2) with musical training may indicate a change in the manner of visual representation of harmonies. We hypothesize that the fusiform gyrus (BA 37), a region used for object recognition, may be associated with a process of basic recognition of the harmonies and harmonic progressions used, while the lingual gyrus (BA 18) may be implicated in higher order harmonic processing. The increased activation found in the subjects without musical training in the anterior cingulate gyrus and left superior frontal gyrus are likely due to increased attention to

$$
X=-50 \quad X=+50
$$

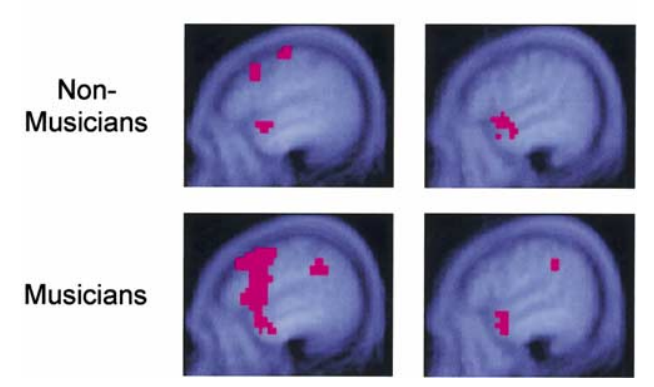

Fig. 1. Functional activation for non-musicians (top) and musicians (bottom) generated by listening to unharmonized melodies vs. listening to random tones. Sagittal slices presented are at $X=-50$ and $X=+50$ (Talairach coordinates). Colored voxels have a corrected $P<0.001$. 


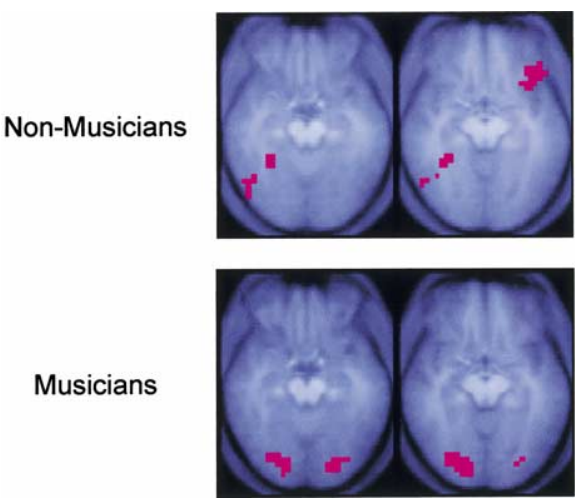

Fig. 2. Functional activation for non-musicians (top) and musicians (bottom) generated by listening to harmonized melodies vs. listening to unharmonized melodies. Axial slices presented are at $Z=-15$ and $Z=$ -10 (Talairach coordinates). Colored voxels have a corrected $P<0.001$.

the auditory stimuli; those regions are active in the musicians during the melody processing task only.

For the musically trained subjects, activation was found in the parietotemporal regions, agreeing with previous results [1] relating activity in these regions to harmonic processing in a subject population which had studied musical instruments since childhood. There are differing hypotheses about the precise role of the parietal lobes in music processing such as auditory working memory [9] and visuo-auditory integration [14]. It is interesting to note that different parietal areas are activated for melodic processing and harmonic processing in the musically trained cohort: melodic processing activates the supramarginal gyrus (BA 40) while harmonic processing activates an area of the intraparietal sulcus near the angular gyrus (BA 39). We hypothesize that these different areas may correspond to different aspects of music processing: the supramarginal gyrus may be used for working memory during melody processing, as the supramarginal gyrus has previously been implicated in visuospatial working memory [6,7], while the angular gyrus may be used for visuospatial processing of harmonies, as the angular gyrus has been shown to be involved in visuospatial tasks [5].

A possible limitation of this study is that each task involves an element of auditory selective attention, due to the scanner noise. This effect was controlled for by using a series of random tones as a control condition for the unharmonized melodies, and by using the unharmonized melodies as a control condition for the harmonized melodies. A previous fMRI study [10] investigating the effects of auditory selective attention for pitch using a dichotic listening paradigm detected activation in posterior parietal regions, as well as processing sites in superior temporal and inferior frontal regions. However, there was little overlap between those regions and the regions detected in the present study, with the exception of a small region in the left inferior frontal gyrus. Thus, we do not expect auditory selective attention to be a major component of the differences in activation patterns.
In conclusion, we have been able to show the recruitment of different neural networks for harmony and melody processing in musicians relative to controls using fMRI. Musical training appears to result in the recruitment of additional cortical areas such as the parietal lobes for music processing. Formal musical training will impact on the listener's processing of the auditory input because he or she will recognize distinctive features such as intervals (e.g. major third, perfect fourth), harmonic types (e.g. major, minor, diminished) and standard harmonic progressions (e.g. I-IV-V-I). Musicians would also be expected to be more proficient at the integration of all the different musical elements being processed. Further research will be necessary in order to elucidate the precise cortical areas recruited by these elements of music processing in trained musicians. We also note that the results of the present study do not disprove the alternative hypothesis that the differing neural circuitry found in musicians has been already established at an early age, prior to musical training, and that the results of our study are simply the results of self-selection bias. Further study involving young children prior to and during musical training will be necessary in order to more clearly delineate the effects of musical training on neural circuitry during the developmental period.

\section{References}

[1] R. Beisteiner, M. Erdler, D. Mayer, A. Gartus, V. Edward, T. Kaindl, S. Golaszewski, G. Lindinger, L. Deecke, A marker for differentiation of capabilities for processing of musical harmonies as detected by magnetoencephalography in musicians, Neurosci. Lett. 277 (1999) $37-40$.

[2] J. Bhattacharya, H. Petsche, Musicians and the gamma band: a secret affair?, NeuroReport 12 (2001) 371-374.

[3] G.C. Crummer, J.P. Walton, J.W. Wayman, E.C. Hantz, R.D. Frisina, Neural processing of musical timbre by musicians, nonmusicians, and musicians possessing absolute pitch, J. Acoust. Soc. Am. 95 (1994) 2720-2727.

[4] K.J. Friston, A.P. Holmes, K.J. Worsley, How many subjects constitute a study?, Neuroimage 10 (1999) 1-5.

[5] S. Gobel, V. Walsh, M.F. Rushworth, The mental number line and the human angular gyrus, Neuroimage 14 (2001) 1278-1289.

[6] M.F. Haberecht, V. Menon, I.S. Warsofsky, C.D. White, J. DyerFriedman, G.H. Glover, E.K. Neely, A.L. Reiss, Functional neuroanatomy of visuo-spatial working memory in Turner syndrome, Hum. Brain Mapp. 14 (2001) 96-107.

[7] H. Kwon, V. Menon, S. Eliez, I.S. Warsofsky, C.D. White, J. DyerFriedman, A.K. Taylor, G.H. Glover, A.L. Reiss, Functional neuroanatomy of visuospatial working memory in fragile $\mathrm{X}$ syndrome: relation to behavioral and molecular measures, Am. J. Psychiatry 158 (2001) 1040-1051.

[8] M. Piccirilli, T. Sciarma, S. Luzzi, Modularity of music: evidence from a case of pure amusia, J. Neurol. Neurosurg. Psychiatry 69 (2000) 541-545.

[9] H. Platel, C. Price, J.C. Baron, R. Wise, J. Lambert, R.S. Frackowiak, B. Lechevalier, F. Eustache, The structural components of music perception. A functional anatomical study, Brain 120 (1997) 229-243.

[10] K.R. Pugh, B.A. Shaywitz, S.E. Shaywitz, R.K. Fulbright, D. Byrd, P. Skudlarski, D.P. Shankweiler, L. Katz, R.T. Constable, J. Fletcher, C. 
Lacadie, K. Marchione, J.C. Gore, Auditory selective attention: an fMRI investigation, Neuroimage 4 (1996) 159-173.

[11] J. Russeler, E. Altenmuller, W. Nager, C. Kohlmetz, T.F. Munte, Event-related brain potentials to sound omissions differ in musicians and non-musicians, Neurosci. Lett. 308 (2001) 33-36.

[12] M. Satoh, K. Takeda, K. Nagata, J. Hatazawa, S. Kuzuhara, Activated brain regions in musicians during an ensemble: a PET study, Brain Res. Cogn. Brain Res. 12 (2001) 101-108.

[13] V.J. Schmithorst, B.J. Dardzinski, S.K. Holland, Simultaneous correction of ghost and geometric distortion artifacts in EPI using a multiecho reference scan, IEEE Trans. Med. Imaging 20 (2001) $535-539$.
[14] J. Sergent, Mapping the musician brain, Hum. Brain Mapp. 1 (1993) $20-38$.

[15] J. Talairach, P. Tournoux, Co-Planar Stereotaxic Atlas of the Human Brain, Thieme, New York, 1988.

[16] P. Thevenaz, M. Unser, A pyramid approach to subpixel registration based on intensity, IEEE Trans. Image Proc. 7 (1998) 27-41.

[17] K.J. Worsley, K.J. Friston, Analysis of fMRI time-series revisited again, Neuroimage 2 (1995) 173-181.

[18] J. Xiong, J.-H. Gao, J.L. Lancaster, P.T. Fox, Clustered pixels analysis for functional MRI activation studies of the human brain, Hum. Brain Mapp. 3 (1995) 287-301. 